Communications in Physics, Vol. 17, No. 4 (2007), pp. 193-197

\title{
QUANTIZATION STRUCTURE OF ORBITAL OSCILLATORS AND MASS SPECTRUM OF BOSONIC STRING
}

\author{
DAO VONG DUC \\ Institute of Physics and Electronics, VAST \\ PHUONG THI THUY HANG \\ Hanoi University of Education
}

\begin{abstract}
We consider a mechanism for removing tachyon field in bosonic string based on some modification of the structure of the commutation relations between coordinate orbital oscillators. This change causes the shifting of the mass spectrum for the component fields of the string field functional.
\end{abstract}

\section{INTRODUCTION}

The existence of tachyon having negative squared mass has been a longstanding problem in string theory [1-4]. The tachyon could be removed in Neveu-Schwarz superstring sector by means of GSO projection operator [5], but remains untouched in bosonic string.

The aim of our work is to consider a model of bosonic string based on a generalized extended form of commutation relations between coordinate oscillators. It turns out that the extra- term in the commutators could serve as a mechanism for shifting the whole mass spectrum and as a consequence the former tachyon gains an additional amount of squared mass to become non- tachyon field. This extra- term at the same time causes some change in the equations of motion for component fields of string field functional.

This paper is organized as follows. In Sec. 2 we consider the algebraic structure of the commutators for quantized orbital string oscillators. On this base the anomaly term of Virasoro algebra is calculated. Its change is responsible for the shifting of mass spectrumSec. 3 is devoted to the BRST charge and the equations of motion within the framework of BRST formalism [6,7].

\section{COMMUTATION RELATIONS FOR ORBITAL OSCILLATORS}

Suppose the orbital oscillators $\alpha_{n}^{\mu}$ in the mode expansion of string coordinate function $X^{\mu}(\tau, \sigma)$,

$$
X^{\mu}(\tau, \sigma)=X^{\mu}+\alpha_{0}^{\mu} \dot{\tau}-i \sum_{n \neq 0} \frac{1}{n} \alpha_{n}^{\mu} e^{i n . \tau} \cos n \sigma
$$

obey the commutation relation of the form:

$$
\left.\left[\alpha_{n}^{\mu}, \alpha_{m}^{\nu}\right]=[f(n)] \eta^{\mu \nu}+g(n) G^{\mu \nu}\right] \dot{\delta}_{n,-m}
$$


for any $n, m \in z$ where $f(n)$ and $g(n)$ are some functions of $n$ having the property:

$$
f(-n)=-f(n), \quad g(-n)=g(n)
$$

$\eta^{\mu \nu}$ is Minkowski metric of space-time, $G^{\nu \mu}$ is some anti symmetric constant tensor $G^{\nu \mu}=$ $-G^{\nu \mu}$.

Without loss of generality we can put $g(0)=1$, and Eq. (2) then give

$$
\left[\alpha_{0}^{\mu}, \alpha_{0}^{\nu}\right]=G^{\mu \nu}
$$

Due to this relation we cannot anymore indentify at with the string momentum $p_{\mu}$. However, we can put

$$
\alpha_{0}^{\mu}=p^{\mu}+\pi^{\mu} ; \quad p^{\mu} \equiv-i \partial^{\mu}
$$

together with the commutation relations

$$
\left[p^{\mu}, \pi^{\nu}\right]=0 ; \quad\left[\pi^{\mu}, \pi^{\nu}\right]=G^{\mu \nu}
$$

Let us consider the Virasoro generators of the form,

$$
L_{n} \equiv d \sum_{k \in Z}: \alpha_{-k}^{\mu} \alpha_{\mu, k+n}:
$$

$d$ being some coefficient constant. Here we adopt the convention that $\pi^{\mu}$ (together with $\left.\alpha_{n}^{\mu}, n>0\right)$ acts as annihilation operator, namely

$$
\pi^{\mu}|0\rangle=0
$$

For arbitrary $n \in z$ we have:

$$
L_{n}|0\rangle=d\left\{\alpha_{0}^{\mu} \alpha_{\mu, n}+\sum_{k=1}^{\infty} \alpha_{-k}^{\mu} \alpha_{\mu, k+n}\right\}|0\rangle
$$

It follows from Eqs. (8) and (9) that:

$$
L_{n}|0\rangle=d p^{2}|0\rangle
$$

and

$$
\begin{gathered}
L_{n}|0\rangle=0 \\
L_{-n}|0\rangle=d\left\{2 p^{\mu} \alpha_{\mu,-n}+\sum_{k=1}^{n-1} \alpha_{\mu, k-n}\right\}|0\rangle
\end{gathered}
$$

for $n>0$.

Now we proceed to the commutation relations for $L_{n}$.

From Eqs. (2) and (7) we have:

$$
\left[L_{n}, \alpha_{m}^{\nu}\right]=2 d\left\{-f(m) \alpha_{n+m}^{\nu}+g(m) G^{\mu \nu} \alpha_{\mu, n+m}\right\}
$$

and

$$
\begin{aligned}
{\left[L_{n}, L_{m}\right]=2 d^{2} \sum_{k \in z}\left\{[f(k+n)-f(k+m)] \alpha_{-k}^{\mu} \alpha_{\mu, k+n+m}\right.} \\
\left.+[g(k+n)-g(k+m)] G_{\mu \nu} \alpha_{-k}^{\mu} \alpha_{k+n+m}^{\nu}\right\}
\end{aligned}
$$


From Eq. (14) we see that for $L_{n}$ to form a closed algebra, $f(n)$ must be of the linear form,

$$
f(n)=c n
$$

and $g(n)$ must be independent of $n$, so that

$$
g(n)=g(0)=1
$$

In this case, by putting $c d=\frac{1}{2}$ we have:

$$
\left[L_{n}, L_{m}\right]=(n-m) L_{n+m}
$$

for $n+m \neq 0$.

In general we can write

$$
\left[L_{n}, L_{m}\right]=(n-m) L_{n+m}+A(n) \delta_{n,-m}
$$

where the anomaly term $A(n)$ can be calculated as follows.

From Eqs. (12), (11), and (18) we have for $n>0$ :

$$
A(n)=\left\langle 0\left|L_{n} L_{-n}\right| 0\right\rangle-2 d n p^{2}
$$

Further calculations with the use of Eqs. (2) and (??) give:

$$
\left\langle 0\left|L_{n} L_{-n}\right| 0\right\rangle=\frac{D}{12} n\left(n^{2}-1\right)+2 d^{2} n G^{2}+2 d n p^{2}
$$

where $G^{2} \equiv G_{\mu \nu} G^{\mu \nu}$.

Hence,

$$
A(n)==\frac{D}{12} n\left(n^{2}-1\right)+2 d^{2} n G^{2}
$$

\section{BRST CHARGE. EQUATIONS OF MOTION}

The BRST charge is constructed according to the formula:

$$
Q=\sum_{n \in z} L_{n} c_{-n}+\frac{1}{2} \sum_{n, m \in Z}(n-m): c_{-n} c_{-m} b_{n+m}:-a c_{0}
$$

where $c_{n}, b_{n}$ are ghost and antighost oscillators obeying the anticommutation rule:

$$
\begin{aligned}
& \left\{c_{n}, b_{m}\right\}=\delta_{n,-m} \\
& \left\{b_{n}, b_{m}\right\}=\left\{c_{n}, c_{m}\right\}=0
\end{aligned}
$$

and

$$
\begin{array}{r}
c_{n}^{+}=c_{-n}, \quad b_{n}^{+}=b_{-n} \\
c_{n}|0\rangle=0, \quad b_{n}|0\rangle=0, \quad n \in Z^{+}
\end{array}
$$

$a$ is the Regge intercept parameter, which is to be chosen such that the nilpotency condition $Q^{2}=0$ is satisfied.

From Eqs. (18), (21)-(23) it can be shown that

$$
Q^{2}=\frac{1}{12} \sum_{n=1}^{\infty}\left\{2-D+24\left(d^{2} G^{2}+a\right)+(D-26) n^{2}\right\} n c_{-n} c_{n}
$$


Hence, the nilpotency of $Q$ requires $D=26$ as before, and

$$
a=1-d^{2} G^{2}
$$

Now let us proceed to the BRST equation

$$
Q \Phi[X(\tau, \sigma)]=0
$$

for the string field functional

$$
\Phi[X(\tau, \sigma)]=\sum_{r=0}^{\infty} \frac{(-i)^{r}}{r !} \phi_{\mu_{1} \mu_{2} \ldots \mu_{r}}^{n_{1} n_{2} \ldots n_{r}}(x) \alpha_{n_{1}}^{\mu_{1}+} \ldots \alpha_{n_{r}}^{\mu_{r}+}|0\rangle, \quad n_{1}, n_{2}, \ldots>0
$$

Eqs. (22), (25) and (26) lead to the following equations:

$$
\begin{gathered}
\left(L_{0}-1+d^{2} G^{2}\right) \Phi[X(\tau, \sigma)]=0 \\
L_{n} \Phi[X(\tau, \sigma)]=0, \quad n>0
\end{gathered}
$$

Inserting the explicit expression of $L_{0}$,

$$
L_{0}=d\left(-\square+2 p^{\mu} \pi_{\mu}+\pi^{2}+2 \sum_{k=1}^{\infty} \alpha_{-k}^{\mu} \alpha_{\mu, k}\right)
$$

into (28) and taking Eqs.(2) and (8) into account, we can derive the following equation for the component fields:

$$
\left\{\square+M^{2}(n)\right\} \phi_{\mu_{1} \mu_{2} \ldots \mu_{r}}^{n_{1} n_{2} \ldots n_{r}}(x)
$$

where

$$
\begin{gathered}
M^{2}(n)=2\left(-1+d^{3} G^{2}+\sum_{k=1}^{r} n_{k}\right) . \\
j_{\mu_{1} \mu_{2} \ldots \mu_{r}}^{n_{1} n_{2} \ldots n_{r}}(x)=2 G_{\mu_{1} \nu_{1} \ldots G_{\mu_{r} \nu_{r}} \phi^{n_{1} n_{2} \ldots n_{r}, \nu_{1} \nu_{2} \ldots \nu_{r}}(x)}
\end{gathered}
$$

Equations (31) and (32) tell that the squared mass of each component field is shifted by an amount $2 d^{2} G^{2}$ as compared to that in conventional theory.

Let us be interested in the low excited modes in the expansion (27) to write:

$$
\Phi[X(\tau, \sigma)]=\left\{\phi(x)-i A_{\mu}(x) \alpha_{-1}^{\mu}-i V_{\mu}(x) \alpha_{-2}^{\mu}-\frac{1}{2} l_{\mu \nu}(x) \alpha_{-1}^{\mu} \alpha_{-1}^{\nu}+\ldots\right\}|0\rangle
$$

Equations (31) and (32) give:

$$
\begin{aligned}
& \left(\square-2+2 d^{2} G^{2}\right) \phi(x)=0 \\
& \left(\square+2 d^{2} G^{2}\right) A_{\mu}(x)=2 G_{\mu \nu} A^{\nu}(x) \\
& \left(\square+2+2 d^{2} G^{2}\right) V_{\mu}(x)=2 G_{\mu \nu} V^{\nu}(x) \\
& \left(\square+2+2 d^{2} G^{2}\right) l_{\mu \nu}(x)=2 G_{\mu \rho} G_{\nu \sigma} l^{\rho \sigma}(x)
\end{aligned}
$$

Note that the first component field $\phi(x)$ corresponding to the tachyon in conventional theory has the squared mass

$$
m_{\phi}^{2}=2\left(d^{2} G^{2}-1\right)
$$


which becomes non-negative if $G^{2} \leq \frac{1}{d^{2}}$.

Eq. (29) lead to the following complementary conditions:

$$
\begin{aligned}
& \partial^{\mu} A_{\mu}(x)=\frac{1}{2} G_{\mu \nu} F^{\mu \nu}(x) \\
& \left(\partial^{\nu}-2 d G^{\nu \sigma} \partial_{\sigma}\right) l_{\mu \nu}(x)=2\left(V_{\mu}-d G_{\mu \nu} V^{\nu}\right) \\
& \partial^{\mu} V_{\mu}(x)-\frac{d}{2} G_{\mu \nu} V^{\mu \nu}(x)=\left(2 d G^{\sigma \mu} G_{\sigma \nu}-\frac{1}{8 d} \delta_{\nu}^{\mu}\right) l_{\mu}^{\nu}
\end{aligned}
$$

where

$$
\begin{aligned}
& F^{\mu \nu}(x) \equiv \partial^{\mu} A^{\nu}(x)-\partial^{\nu} A^{\mu}(x) \\
& V^{\mu \nu}(x) \equiv \partial^{\mu} V^{\nu}(x)-\partial^{\nu} V^{\mu}(x)
\end{aligned}
$$

\section{ACKNOWLEDGMENTS}

The authors are grateful to their colleagues at Hanoi lnstitude of Physics and Hanoi University of Education for the interest in this work.

\section{REFERENCES}

[1] M. B. Green, J. H. Schwarz, E. Witten, Superstring Theory, Cambridge 1987.

[2] L. Brink, M. Henneaux, Principles of String Theory, New York 1988.

[3] M. Kaku, Introduction to Superstring Theory, World Scientific 1989.

[4] L. Brink, D. Friedan, A. M. Polyakov, Physics and Mathematics of Strings, World Scientific 1990.

[5] F. Gliozzi, J. Scherk, D. Olive, Nucl. Phys. B 122(1977) 253.

[6] C. Becchi, A. Rouet, R. Stora, Ann. Phys. 98 (1976) 287.

[7] U Dao Vong Duc, Nguyen Thi Hong, J. Phys. A 23 (1990) 1.

Received 31 May $200 \%$. 
